\title{
Link colorings and the Goeritz matrix
}

\author{
Lorenzo Traldi \\ Lafayette College \\ Easton, Pennsylvania 18042
}

\begin{abstract}
We discuss the connection between colorings of a link diagram and the Goeritz matrix.

Keywords. Coloring, Goeritz matrix, link

Mathematics Subject Classification. 57M25, 05C10, 05C22, 05C50
\end{abstract}

\section{Introduction}

This paper is inspired by two papers that have appeared previously, the first written by Nanyes 20 and the second written by Lamey, Silver and Williams 15. These papers involve the connection between two ideas from classical knot theory, the Goeritz matrix and colorings of link diagrams. Goeritz introduced his matrix in 1933 [7, and it was also discussed in Reidemeister's classic treatise 22. The Goeritz matrix has attracted the attention of many researchers over the decades; see [3, 4, 8, 9, 10, 11, 14, 16, 17, 18, 21, 23, 25] for instance. Link colorings were mentioned in the textbook of Crowell and Fox [5. Exercises VI. 6 and VI. 7]. Link colorings can be defined easily and they provide very simple nontriviality proofs for some knots and links, so it is natural that they are mentioned in many introductory discussions of knot theory, like [1, 2, 13, 19].

Crowell and Fox used link colorings for the purpose of providing combinatorial descriptions of certain kinds of representations of link groups (the fundamental groups of link complements in $\mathbb{S}^{3}$ ). At first glance, this purpose does not suggest a connection with the Goeritz matrix; link groups are nonabelian in general, and we would expect a matrix of integers to be associated with abelian groups instead. Nanyes [20] provided an indirect connection: link colorings with values in an abelian group $A$ are connected with representations of link groups in a semidirect product of $A$ and $\mathbb{Z} / 2 \mathbb{Z}$, and these representations in turn are connected with the Goeritz matrix. Nanyes's discussion is quite general; it applies to any link diagram, and any abelian group. His presentation requires the theory connecting group representations to covering spaces, and the fact that the Goeritz matrix is associated with 2 -fold coverings of $\mathbb{S}^{3}$ branched over links [14, 16, 23].

Extending a theme established earlier by Kauffman 12 and Carter, Silver and Williams 2, Lamey, Silver and Williams [15] showed that the colorings 
introduced by Crowell and Fox are related to other types of link colorings, and they observed that one of these other types of link colorings is directly related to the Goeritz matrix. Unlike Nanyes, they restricted their attention to colorings with values in a field, and to alternating link diagrams for which one of the associated checkerboard graphs is connected. This portion of their paper does not require results from algebraic topology; the arguments involve only relatively elementary properties of matrices and plane graphs.

Taken together, the papers of Lamey, Silver and Williams [15] and Nanyes 20] suggest a problem of exposition: to provide an explanation of the connection between link colorings and the Goeritz matrix that is as direct and elementary as the discussion in [15], and as general as the discussion in [20. The purpose of the present paper is to provide such an explanation.

Before starting, we should thank an anonymous reviewer whose good advice improved the exposition in several regards.

\section{Link colorings}

We use link diagrams to represent links in the usual way. A tame, classical link diagram begins with a finite number of piecewise smooth, simple closed curves $\gamma_{1}, \ldots, \gamma_{\mu}$ in the plane. The only (self-)intersections of these curves are transverse double points, called crossings; and there are only finitely many of these. At each crossing a very short segment of one of the incident curves is removed. The resulting piecewise smooth 1-dimensional subset of $\mathbb{R}^{2}$ is a link diagram. The set of arc components of a link diagram $D$ is denoted $A(D)$. The faces of $D$ are the arc components of $\mathbb{R}^{2}-\cup \gamma_{i}$, and the set of faces of $D$ is denoted $F(D)$. A link diagram $D$ represents a link $L(D)$ in $\mathbb{R}^{3}$, which consists of piecewise smooth, simple closed curves $K_{1}, \ldots, K_{\mu}$ such that $K_{i}$ projects to $\gamma_{i}$ for each $i . K_{1}, \ldots, K_{\mu}$ are the components of $L(D)$. The removal of segments in $D$ is used to distinguish the underpassing arc at each crossing.

A link diagram is split if the union $\cup \gamma_{i}$ is not connected. In keeping with the goal of generality mentioned in the introduction, our discussion includes split diagrams as well as non-split diagrams.

We use $A$ to denote an arbitrary abelian group. To avoid notational confusion with the arcs of a link diagram, we usually use $\alpha$ to represent an element of $A$.

Definition 1 If $D$ is a link diagram then a Fox coloring of $D$ with values in an abelian group $A$ is a mapping $f: A(D) \rightarrow A$ with the following property.

- If there is a crossing of $D$ at which the underpassing arcs are $a_{1}$ and $a_{2}$ and the overpassing arc is $a_{3}$, then $f\left(a_{1}\right)+f\left(a_{2}\right)=2 \cdot f\left(a_{3}\right)$.

The set of Fox colorings of $D$ with values in $A$ is denoted $\mathcal{F}_{A}(D)$.

Notice that we do not require a Fox coloring of $D$ to be 0 on any arc of $D$. This generality gives $\mathcal{F}_{A}(D)$ a couple of pleasant (and obvious) naturality 
properties, which do not hold in 22, 15, 20. Suppose $D$ is the split union of subdiagrams $D_{1}$ and $D_{2}$, i.e., $D=D_{1} \cup D_{2}$ and no crossing of $D$ involves both $D_{1}$ and $D_{2}$. Then the union of functions defines a bijective map

$$
\mathcal{F}_{A}\left(D_{1}\right) \times \mathcal{F}_{A}\left(D_{2}\right) \rightarrow \mathcal{F}_{A}(D)
$$

for every abelian group $A$, and an injective map

$$
\mathcal{F}_{A_{1}}\left(D_{1}\right) \times \mathcal{F}_{A_{2}}\left(D_{2}\right) \rightarrow \mathcal{F}_{A_{1} \oplus A_{2}}(D)
$$

for every pair of abelian groups $A_{1}, A_{2}$.

Definition 2 Let $D$ be a link diagram. Then a Dehn coloring of $D$ with values in an abelian group $A$ is a mapping $d: F(D) \rightarrow A$ with the following property.

- Suppose $F$ and $F^{\prime}$ are two faces of $D$ whose boundaries share a segment of positive length, contained in an arc $a \in A(D)$. Then the sum $d(F)+d\left(F^{\prime}\right)$ depends only on a.

The set of Dehn colorings of $D$ with values in $A$ is denoted $\mathcal{D}_{A}(D)$.

Definition 2 gives rise to one equation for each crossing of $D$. If the faces incident at a crossing are indexed as in Figure 1 then the boundaries of $F_{1}$ and $F_{4}$ share a segment of positive length contained in $a_{3}$, and so do the boundaries of $F_{2}$ and $F_{3}$. Consequently Definition 2 requires $d\left(F_{1}\right)+d\left(F_{4}\right)=d\left(F_{2}\right)+d\left(F_{3}\right)$.

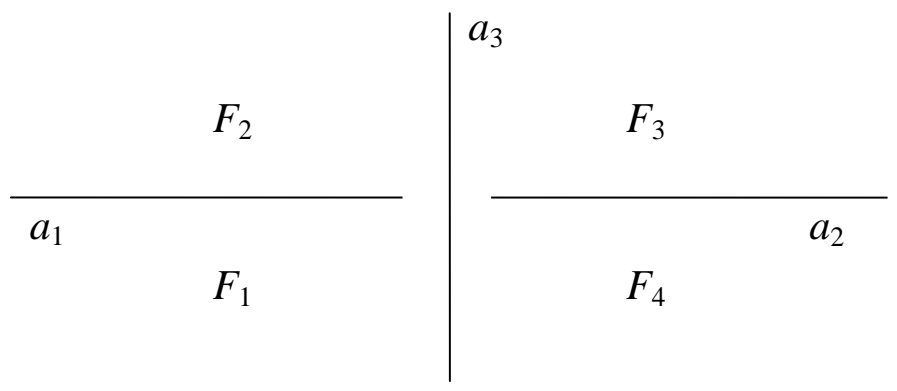

Figure 1: The arcs and faces incident at a crossing.

We should remark that the term "Dehn coloring" indicates a courteous regard for one of the important early contributors to combinatorial group theory and geometric topology, but it does not indicate that these colorings were actually introduced by Dehn. Definition 2 was mentioned by Kauffman 12 and developed further by Carter, Silver and Williams [2], who chose the name "Dehn coloring" because these colorings are connected with a way to present link groups that was introduced by Dehn. We should also remark that like Definition 1. Definition 2 is generalized from [2, 12] - we allow $A$ to be an arbitrary abelian group, and we do not require any value of a Dehn coloring to be 0 .

As discussed in 2] and [12, Dehn colorings and Fox colorings are closely related to each other. 
Definition 3 Let $D$ be a link diagram, and $A$ an abelian group. Then $\mathcal{D}_{A}(D)$ and $\mathcal{F}_{A}(D)$ are both abelian groups under pointwise addition of functions. There is a homomorphism $\varphi: \mathcal{D}_{A}(D) \rightarrow \mathcal{F}_{A}(D)$, defined by: if $d \in \mathcal{D}_{A}(D)$ then

$$
\varphi(d)(a)=d\left(F_{1}\right)+d\left(F_{2}\right)
$$

whenever $F_{1}, F_{2}$ are two faces of $F$ whose boundaries share a segment of positive length on $a$.

Definition 2 implies that $\varphi(d)$ is well defined, and also that $\varphi(d)$ satisfies Definition 1 with arcs and faces indexed as in Figure 1.

$$
\begin{aligned}
\varphi(d)\left(a_{1}\right)+\varphi(d)\left(a_{2}\right) & =d\left(F_{1}\right)+d\left(F_{2}\right)+d\left(F_{3}\right)+d\left(F_{4}\right) \\
& =\left(d\left(F_{1}\right)+d\left(F_{4}\right)\right)+\left(d\left(F_{2}\right)+d\left(F_{3}\right)\right)=2 \cdot \varphi(d)\left(a_{3}\right) .
\end{aligned}
$$

As is well known, the faces of a link diagram can be colored in a checkerboard fashion, so that whenever the boundaries of two faces share a segment of positive length, one face is shaded and the other is not shaded. It is traditional to prefer one of the two possible checkerboard shadings, by specifying whether the unbounded face should be shaded or unshaded. In keeping with our theme of generality, however, we do not follow this tradition. The gain in generality is vacuous at this point, but later it will mean that the two different shadings of a link diagram give rise to two different Goeritz matrices. The theory we develop will apply equally well to both matrices.

Arbitrarily choose one of the two checkerboard shadings of a link diagram $D$, and let $\sigma: F(D) \rightarrow\{0,1\}$ be the map defined as follows.

$$
\sigma(F)= \begin{cases}0, & \text { if } F \text { is unshaded } \\ 1, & \text { if } F \text { is shaded }\end{cases}
$$

If $\alpha, \beta \in A$, then $D$ has a Dehn coloring $d_{\alpha, \beta}$ given by

$$
d_{\alpha, \beta}(F)=(1-\sigma(F)) \cdot \alpha+\sigma(F) \cdot \beta .
$$

This mapping satisfies Definition 2 because in Figure 1 each of the pairs $\left\{F_{1}, F_{4}\right\}$, $\left\{F_{2}, F_{3}\right\}$ includes one shaded face and one unshaded face, so that

$$
d_{\alpha, \beta}\left(F_{1}\right)+d_{\alpha, \beta}\left(F_{4}\right)=\alpha+\beta=d_{\alpha, \beta}\left(F_{2}\right)+d_{\alpha, \beta}\left(F_{3}\right) .
$$

The next result is our version of [2, Theorem 2.2]. We have an epimorphism rather than an isomorphism because our Dehn colorings are not required to be 0 anywhere. The proof is essentially the same as in [2], but we provide details for the reader's convenience.

Theorem 4 The homomorphism $\varphi: \mathcal{D}_{A}(D) \rightarrow \mathcal{F}_{A}(D)$ is surjective, and $\operatorname{ker} \varphi$ $=\left\{d_{\alpha,-\alpha} \mid \alpha \in A\right\}$. 
Proof. Suppose $f \in \mathcal{F}_{A}(D)$, and $F_{0}$ is some face of $D$. Choose an arbitrary element $\alpha_{0} \in A$, and define $d\left(F_{0}\right)=\alpha_{0}$. For any other face $F$ of $D$, choose a smooth path $P$ from a point in $F_{0}$ to a point in $F$. We may presume that $P$ does not come close enough to any crossing to intersect any of the short segments removed to indicate undercrossings, and we may also presume that there are only finitely many intersections between $P$ and $D$. Suppose that as we follow $P$ from $F_{0}$ to $F$, we encounter faces and arcs in the order $F_{0}, a_{1}^{\prime}, F_{1}^{\prime}, \ldots, a_{k}^{\prime}, F_{k}^{\prime}=F$. Then we define

$$
d(F)=(-1)^{k} \alpha_{0}+\sum_{i=1}^{k}(-1)^{k-i} f\left(a_{i}^{\prime}\right)
$$

It turns out that $d(F)$ is independent of the path $P$. To see why, suppose $P^{\prime}$ is some other smooth path from $F_{0}$ to $F$. Then $P$ can be smoothly deformed into $P^{\prime}$. When a smooth deformation does not involve any crossing of $D$, there is no effect on $d(F)$. When the deformation passes through a crossing, the effect is to replace one passage of an $\operatorname{arc}$ of $D$ with three passages. For instance, in Figure 1 we might replace a passage across $a_{1}$ from $F_{1}$ to $F_{2}$ with a sequence of three passages; the first from $F_{1}$ to $F_{4}$ across $a_{3}$, the second from $F_{4}$ to $F_{3}$ across $a_{2}$, and the third from $F_{3}$ to $F_{2}$ across $a_{3}$. Suppose the original passage from $F_{1}$ to $F_{2}$ was indexed with $F_{1}=F_{j}^{\prime}, a_{1}=a_{j+1}^{\prime}$ and $F_{2}=F_{j+1}^{\prime}$. The value given by (1) is not changed because Definition 1 tells us that

$$
\begin{aligned}
& (-1)^{k-j} f\left(a_{3}\right)+(-1)^{k-j-1} f\left(a_{2}\right)+(-1)^{k-j-2} f\left(a_{3}\right) \\
& =(-1)^{k-j} \cdot\left(-f\left(a_{2}\right)+2 \cdot f\left(a_{3}\right)\right)=(-1)^{k-j} f\left(a_{1}\right) .
\end{aligned}
$$

The same kind of argument holds if any other one of the passages in Figure 1 is replaced with the remaining three.

By the way, "replacing one passage with three" might seem to indicate that the path is getting longer. This is not true in general because some of the three new passages may be canceled if $P$ includes the opposite passages through the same crossings. Also, if $i<j$ and $F_{i}^{\prime}=F_{j}^{\prime}$ then the argument above shows that the value of (1) is not changed if $P$ is shortened to a path corresponding to the list $F_{0}, a_{1}^{\prime}, F_{1}^{\prime}, \ldots, a_{i}^{\prime}, F_{i}^{\prime}, a_{j+1}^{\prime}, \ldots, F_{k}^{\prime}=F$.

To verify that $d \in \mathcal{D}_{A}(D)$, suppose the $d$ values of the four faces indicated in Figure 1 are defined using a path $P$ from $F$ that enters the figure in the lower left hand corner. Then equation (1) implies that $d\left(F_{2}\right)=f\left(a_{1}\right)-d\left(F_{1}\right)$, $d\left(F_{3}\right)=f\left(a_{3}\right)-d\left(F_{2}\right)$ and $d\left(F_{4}\right)=f\left(a_{3}\right)-d\left(F_{1}\right)$. It follows that

$$
d\left(F_{1}\right)+d\left(F_{4}\right)=f\left(a_{3}\right)=d\left(F_{2}\right)+d\left(F_{3}\right) .
$$

As $\varphi(d)=f$, we have verified that $\varphi$ is surjective. The description of $\operatorname{ker} \varphi$ in the statement is obvious.

It is easy to see that the epimorphism $\varphi$ splits.

Theorem 5 If $D$ is a link diagram and $A$ is an abelian group then $\mathcal{D}_{A}(D)$ is the internal direct sum of $\operatorname{ker} \varphi$ and a subgroup isomorphic to $\mathcal{F}_{A}(D)$. 
Proof. Let $F_{0}$ be a fixed but arbitrary face of $D$. Let $\delta: \mathcal{F}_{A}(D) \rightarrow \mathcal{D}_{A}(D)$ be the function defined by the construction in the proof of Theorem 4, always using $\alpha_{0}=0$. Then formula (1) tells us that $\delta$ is a homomorphism.

As $\varphi \delta(f)=f \forall f \in \mathcal{F}_{A}(D)$, the theorem follows.

\section{The Goeritz matrix}

Suppose $D$ is a link diagram, and $s$ is one of the two checkerboard colorings of its faces. Then each crossing of $D$ is assigned a Goeritz index $\eta \in\{-1,1\}$ as indicated in Figure 2 That is, $\eta=1$ if the overpassing arc appears on the right-hand sides of the unshaded face(s) incident at the crossing, and $\eta=-1$ if the overpassing arc appears on the left-hand sides of the unshaded face(s) incident at the crossing. Equivalently, if we index faces as in Figure 1 then $\eta=(-1)^{\sigma\left(F_{4}\right)}$.

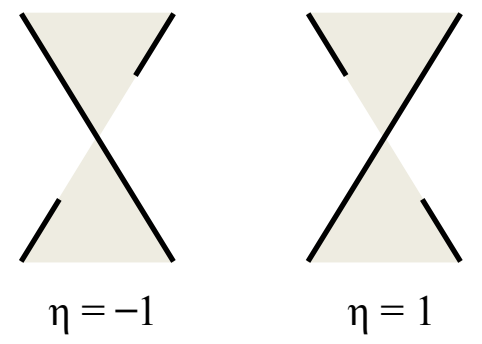

Figure 2: The Goeritz index of a crossing.

Definition 6 Let $D$ be a link diagram, and let $s$ be either of the two checkerboard shadings of the faces of $D$. Let $F_{1}, \ldots, F_{n}$ be the unshaded faces of $D$. For $i, j \in\{1, \ldots, n\}$ let $C_{i j}$ be the set of crossings of $D$ incident on $F_{i}$ and $F_{j}$. Then the unreduced Goeritz matrix of $D$ with respect to $s$ is the $n \times n$ matrix $G(D, s)$ with entries defined as follows.

$$
G(D, s)_{i j}= \begin{cases}-\sum_{c \in C_{i j}} \eta(c), & \text { if } i \neq j \\ -\sum_{k \neq i} G(D, s)_{i k}, & \text { if } i=j\end{cases}
$$

Before proceeding we make five remarks about Definition 6. (i) $G(D, s)$ is a symmetric integer matrix, whose rows and columns sum to 0 . It is traditional to remove one row and the corresponding column, to obtain a matrix whose determinant might not be 0 . However we do not follow this tradition here; that is why we call our matrix "unreduced." (ii) $G(D, s)$ ignores any crossing that is incident on only one unshaded face. (iii) There are two checkerboard graphs or Tait graphs associated with $D$. One graph has vertices corresponding to the shaded faces, and the other graph has vertices corresponding to the 
unshaded faces. Both have edges corresponding to the crossings of $D$. The matrix $G(D, s)$ is the Laplacian matrix of the unshaded checkerboard graph, with the Goeritz indices interpreted as edge weights. There is a well developed theory of Laplacian matrices of weighted graphs; the interested reader might consult [6, Chapter 13] for an introduction. (iv) If $\bar{s}$ is the other checkerboard shading of $D$ then the matrices $G(D, s)$ and $G(D, \bar{s})$ may be quite different; for instance, one may be much larger than the other. Nevertheless there is an intimate relationship between the two matrices. See [17] for a discussion. (v) Despite the connection between Goeritz matrices of link diagrams and Laplacian matrices of graphs, we have chosen to use relatively little terminology from graph theory in this paper. One reason for this choice is that the definition of the dual of a plane graph always results in a connected graph. In contrast, the checkerboard graphs of a split link diagram may be disconnected.

We use $A^{n}$ to denote the direct sum of $n$ copies of the abelian group $A$. If $F_{1}, \ldots, F_{n}$ are the unshaded faces of $D$ then $G(D, s)$ defines a homomorphism $A^{n} \rightarrow A^{n}$ of abelian groups. We are interested in the properties of the kernel of this homomorphism.

Definition 7 Let $D$ be a link diagram with a shading $s$ whose unshaded faces are $F_{1}, \ldots, F_{n}$. If $A$ is an abelian group then

$$
\operatorname{ker}_{A} G(D, s)=\left\{v \in A^{n} \mid G(D, s) \cdot v=0\right\} .
$$

That is, $\operatorname{ker}_{A} G(D, s)$ is the subset of $A^{n}$ consisting of elements that are orthogonal to the rows of $G(D, s)$.

The next proposition is concerned with a special property of some split link diagrams: they have unshaded faces whose boundaries are not connected.

Proposition 8 Let $D$ be a link diagram with a shading s whose unshaded faces are $F_{1}, \ldots, F_{n}$. Let $\gamma$ be a simple closed curve, which forms part of the boundary of $F_{i}$. Let $\rho(\gamma) \in \mathbb{Z}^{n}$ be the vector defined as follows.

$$
\rho(\gamma)_{j}= \begin{cases}-\sum_{c \in C_{i j} \cap \gamma} \eta(c), & \text { if } i \neq j \\ -\sum_{k \neq i} \rho(\gamma)_{k}, & \text { if } i=j\end{cases}
$$

Then for any abelian group $A, \rho(\gamma) \cdot v=0 \forall v \in \operatorname{ker}_{A} G(D, s)$.

Proof. If $\gamma$ is the entire boundary of $F_{i}$ then $\rho(\gamma)$ is the $i^{\text {th }}$ row of $G(D, s)$. If $\gamma$ is not incident on any crossing that involves an unshaded face other than $F_{i}$, then $\rho(\gamma)=0$. In either of these cases it is obvious that $\rho(\gamma) \cdot v=0$ $\forall v \in \operatorname{ker}_{A} G(D, s)$.

Suppose $\gamma$ is not the entire boundary of $F_{i}$, and $\gamma$ is incident on some crossing that involves an unshaded face other than $F_{i}$. According to the Jordan curve theorem, $\mathbb{R}^{2}-\gamma$ has two components, one inside $\gamma$ and the other outside $\gamma$. Suppose the other unshaded face that shares a crossing on $\gamma$ with $F_{i}$ lies inside $\gamma$. (See Figure 3 for an example of this sort. In the figure, $\gamma$ is indicated with 


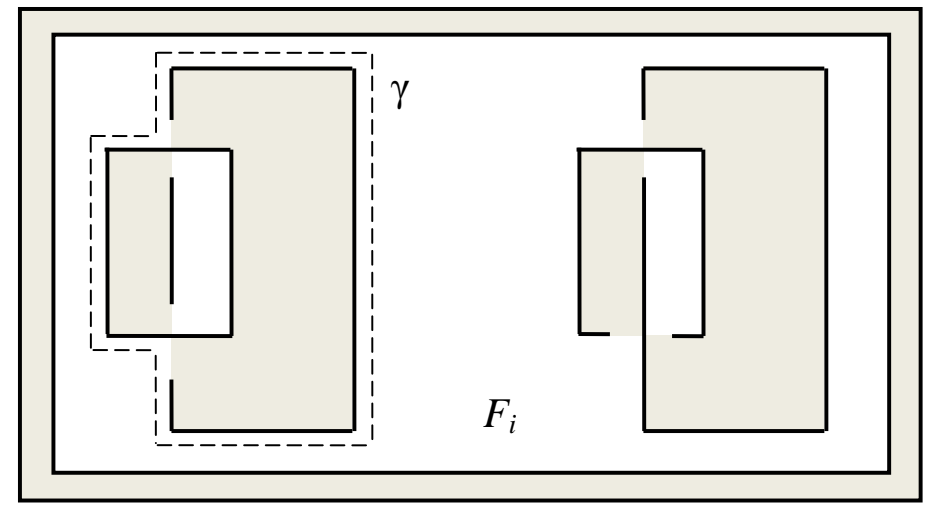

Figure 3: The boundary of $F_{i}$ consists of three closed curves.

dashes; it is displaced a little bit for clarity.) Let $D^{\prime}$ be the subdiagram of $D$ that includes $\gamma$ and all the arcs of $D$ contained inside $\gamma$, and let $s^{\prime}$ be the shading of the faces of $D^{\prime}$ defined by $s$. Then $F_{i}$ corresponds to an unshaded face of $D^{\prime}$, whose boundary in $D^{\prime}$ is $\gamma$. The other unshaded faces of $D^{\prime}$ are the unshaded faces of $D$ contained inside $\gamma$, and if $F_{j}$ is an unshaded face of $D$ contained inside $\gamma$ then the only difference between the row of $G(D, s)$ corresponding to $F_{j}$ and the row of $G\left(D^{\prime}, s^{\prime}\right)$ corresponding to $F_{j}$ is that the former has extra 0 entries in columns corresponding to unshaded faces of $D$ not contained inside $\gamma$.

That is, if $G^{\prime}$ is the submatrix of $G\left(D^{\prime}, s^{\prime}\right)$ obtained by removing the row corresponding to the face of $D^{\prime}$ that contains $F_{i}$, then

$$
G(D, s)=\left(\begin{array}{cc}
G^{\prime} & 0 \\
G^{\prime \prime} & G^{\prime \prime \prime}
\end{array}\right)
$$

for some submatrices $G^{\prime \prime}$ and $G^{\prime \prime \prime}$. Definition 6 makes it clear that the sum of the rows of a Goeritz matrix is 0 ; hence we can obtain the row of $G\left(D^{\prime}, s^{\prime}\right)$ corresponding to the face of $D^{\prime}$ that contains $F_{i}$ by summing the other rows of $G\left(D^{\prime}, s^{\prime}\right)$, and multiplying by -1 . It follows that $-\rho(\gamma)$ is the sum of the rows of $\left(\begin{array}{ll}G^{\prime} & 0\end{array}\right)$, so $\rho(\gamma)$ is an element of the row space of $G(D, s)$. We conclude that $\rho(\gamma) \cdot v=0$ for every $v \in \operatorname{ker}_{A} G(D, s)$.

If the other unshaded face that shares a crossing on $\gamma$ with $F_{i}$ lies outside $\gamma$ then the same argument applies, with "inside $\gamma$ " changed to "outside $\gamma$ " throughout.

Proposition 9 Let $D$ be a link diagram with a shading s whose unshaded faces are $F_{1}, \ldots, F_{n}$. Suppose $A$ is an abelian group, $v=\left(v_{1}, \ldots, v_{n}\right) \in \operatorname{ker}_{A} G(D, s)$, and $F_{i}$ and $F_{j}$ are incident at a crossing where only one shaded face is incident. Then $v_{i}=v_{j}$. 

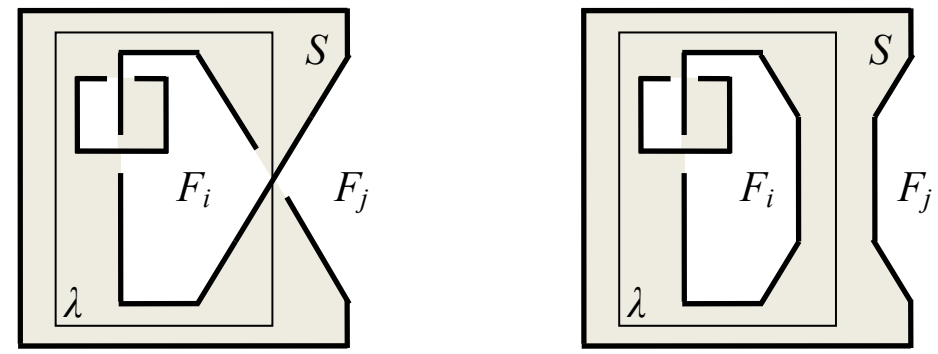

Figure 4: Examples of $D$ and $D^{\prime}$ in Proposition 9 .

Proof. Suppose $F_{i}$ and $F_{j}$ are incident at a crossing $c$, and $S$ is the only shaded face of $D$ incident at $c$. Then there is a piecewise smooth closed curve $\lambda$ that is contained in the interior of $S$ except for the fact that it passes through $c$. Interchanging $i$ and $j$ if necessary, we may presume that $F_{i}$ is contained in the region inside $\lambda$ and $F_{j}$ is contained in the region outside $\lambda$. Let $D^{\prime}$ be the link diagram obtained from $D$ by smoothing $c$ in such a way that $F_{i}$ is detached from $F_{j}$. (See Figure 4 for an example.)

Let $D^{\prime \prime}$ be the subdiagram of $D^{\prime}$ contained inside $\lambda$, and $D^{\prime \prime \prime}$ the subdiagram outside $\lambda$. If $s^{\prime}, s^{\prime \prime}$ and $s^{\prime \prime \prime}$ are the shadings of $D^{\prime}, D^{\prime \prime}$ and $D^{\prime \prime \prime}$ defined by $s$ then the Goeritz matrix of $D^{\prime}$ is

$$
G\left(D^{\prime}, s^{\prime}\right)=\left(\begin{array}{cc}
G\left(D^{\prime \prime}, s^{\prime \prime}\right) & 0 \\
0 & G\left(D^{\prime \prime \prime}, s^{\prime \prime \prime}\right)
\end{array}\right) .
$$

Let $G(D, s)_{i}, G\left(D^{\prime}, s^{\prime}\right)_{i}$ and $G\left(D^{\prime \prime}, s^{\prime \prime}\right)_{i}$ be the rows of $G(D, s), G\left(D^{\prime}, s^{\prime}\right)$ and $G\left(D^{\prime \prime}, s^{\prime \prime}\right)$ corresponding to $F_{i}$, respectively. Also, let $G^{\prime \prime}$ be the submatrix of $G\left(D^{\prime \prime}, s^{\prime \prime}\right)$ obtained by removing $G\left(D^{\prime \prime}, s^{\prime \prime}\right)_{i}$. Then $H=\left(G^{\prime \prime} \quad 0\right)$ is a submatrix of $G(D, s)$, and the sum of the rows of $H$ is the negative of $G\left(D^{\prime}, s^{\prime}\right)_{i}$. Let $w=\left(w_{1}, \ldots, w_{n}\right)$ be the vector whose only nonzero entries are $w_{i}=\eta(c)$ and $w_{j}=-\eta(c)$. Then $w$ is the difference between $G(D, s)_{i}$ and $G\left(D^{\prime}, s^{\prime}\right)_{i}$. It follows that if we add $G(D, s)_{i}$ to the sum of the rows of $H$, we get $w$.

We conclude that $w$ is included in the row space of $G(D, s)$, so $w \cdot v=0$ $\forall v \in \operatorname{ker}_{A} G(D, s)$.

\section{$4 \mathcal{D}_{A}(D)$ and $\operatorname{ker}_{A} G(D, s)$}

In this section we generalize ideas of Lamey, Silver and Williams [15] to the Goeritz matrix. The foundation for this generalization has already been laid: Definitions 1 and 2 allow for colorings with values in arbitrary abelian groups, Definition 6 allows for link diagrams with arbitrary crossing signs, and Propositions 8 and 9 provide useful special properties of link diagrams with disconnected checkerboard graphs. Extending the arguments of [15] to the general setting requires only a little attention to special cases, and one additional idea given in Definition 13 . 
Proposition 10 Let $s$ be a shading of a link diagram $D$, whose unshaded faces are $F_{1}, \ldots, F_{n}$. If $d \in \mathcal{D}_{A}(D)$, then the vector $v(d)=\left(d\left(F_{1}\right), \ldots, d\left(F_{n}\right)\right)$ is an element of $\operatorname{ker}_{A} G(D, s)$.

Proof. Suppose $1 \leq i \leq n$, and let $G(D, s)_{i}$ be the $i^{\text {th }}$ row of $G(D, s)$. The dot product $G(D, s)_{i} \cdot v(d)$ is a sum of contributions from the crossings of $D$ incident on $F_{i}$. To analyze these contributions, consider a crossing of $D$ incident on $F_{i}$ and $F_{j} \neq F_{i}$, pictured in Figure 5 .

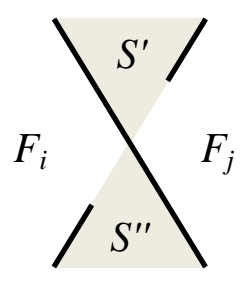

$$
\eta=-1
$$

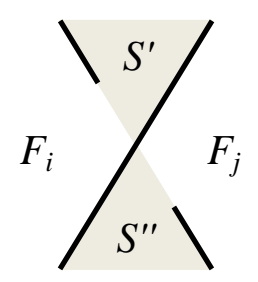

$\eta=1$

Figure 5: A crossing incident on $F_{i}$ and $F_{j}$.

If $\eta=-1$, then the contribution of this crossing to $G(D, s)_{i} \cdot v(d)$ includes two terms: $-\eta \cdot d\left(F_{j}\right)=d\left(F_{j}\right)$ from $G(D, s)_{i j} \cdot d\left(F_{j}\right)$ and $\eta \cdot d\left(F_{i}\right)=-d\left(F_{i}\right)$ from $G(D, s)_{i i} \cdot d\left(F_{i}\right)$. Consulting Definition 2, we see that the contribution of this crossing is

$$
d\left(F_{j}\right)-d\left(F_{i}\right)=d\left(S^{\prime}\right)-d\left(S^{\prime \prime}\right) .
$$

If $\eta=1$, the contribution of this crossing to $G(D, s)_{i} \cdot v(d)$ includes $-\eta \cdot d\left(F_{j}\right)=$ $-d\left(F_{j}\right)$ from $G(D, s)_{i j} \cdot d\left(F_{j}\right)$ and $\eta \cdot d\left(F_{i}\right)=d\left(F_{i}\right)$ from $G(D, s)_{i i} \cdot d\left(F_{i}\right)$. Consulting Definition 2, we see that the contribution of this crossing is

$$
-d\left(F_{j}\right)+d\left(F_{i}\right)=d\left(S^{\prime}\right)-d\left(S^{\prime \prime}\right) .
$$

Either way, the contribution is $d\left(S^{\prime}\right)-d\left(S^{\prime \prime}\right)$.

Now, consider a crossing as pictured in Figure 5 , but with $i=j$. This crossing is ignored by $G(D, s)$, so its contribution to $G(D, s)_{i} \cdot v(d)$ is 0 . On the other hand, Definition 2 tells us that $F_{i}=F_{j} \Longrightarrow d\left(S^{\prime}\right)=d\left(S^{\prime \prime}\right)$, so $d\left(S^{\prime}\right)-d\left(S^{\prime \prime}\right)$ is 0 too.

In every case, then, the contribution of the crossing pictured in Figure 5 to $G(D, s)_{i} \cdot v(d)$ is $d\left(S^{\prime}\right)-d\left(S^{\prime \prime}\right)$. If we follow the boundary component of $F_{i}$ that contains this crossing in the clockwise direction, we see that the total of the contributions of all the crossings is a telescoping sum:

$$
\left(d\left(S^{\prime}\right)-d\left(S^{\prime \prime}\right)\right)+\left(d\left(S^{\prime \prime}\right)-d\left(S^{\prime \prime \prime}\right)\right)+\cdots+\left(d\left(S^{(k)}\right)-d\left(S^{\prime}\right)\right)=0,
$$

where $k$ is the number of crossings incident on this boundary component of $F_{i}$. As every boundary component of $F_{i}$ contributes $0, G(D, s)_{i} \cdot v(d)=0$.

Proposition 10 tells us that $d \mapsto v(d)$ defines a map $v: \mathcal{D}_{A}(F) \rightarrow \operatorname{ker}_{A} G(D, s)$. It is easy to see that $v$ is a homomorphism of abelian groups, and that ker $v$ 
consists of the Dehn colorings that are identically 0 on unshaded faces of $D$. It is more difficult to see another important property of $v$ : it is surjective.

Proposition 11 Let $D$ be a link diagram, with a shading $s$ whose unshaded faces are $F_{1}, \ldots, F_{n}$. Let $A$ be an abelian group, and suppose $v \in \operatorname{ker}_{A} G(D, s)$. Then there is a $d \in \mathcal{D}_{A}(D)$ with $v=v(d)$.

Proof. For each unshaded face $F_{i}$ of $D$, define $d\left(F_{i}\right)$ to be the $i^{\text {th }}$ coordinate of the vector $v$. Our job is to define $d$ values for the shaded faces, in such a way that the resulting function $d: F(D) \rightarrow A$ satisfies Definition 2

Choose any shaded face $S$ of $D$, choose any element $\alpha \in A$, and define $d(S)=\alpha$. Repeat the following recursive step as many times as possible. If $S^{\prime \prime}$ is a shaded face such that $d\left(S^{\prime \prime}\right)$ has not yet been defined, and the boundary of $S^{\prime \prime}$ shares a crossing with the boundary of a shaded face $S^{\prime}$ such that $d\left(S^{\prime}\right)$ has been defined, then define $d\left(S^{\prime \prime}\right)$ to be the unique element of $A$ that satisfies Definition 2 at this crossing.

After the process of the preceding paragraph is completed, there may still be shaded faces whose $d$ values have not been defined; these faces do not share any crossing with shaded faces whose $d$ values have been defined. Go back to the preceding paragraph, and change the first sentence to read "Choose any shaded face $S$ of $D$ whose $d$ value has not yet been defined, choose any element $\alpha \in A$, and define $d(S)=\alpha$." Then repeat the entire process of the preceding paragraph as many times as possible.

We claim that this recursion yields a well defined function $d \in \mathcal{D}_{A}(D)$. The claim certainly holds in case no crossing appears in $D$ as $G(D, s)$ is the 0 matrix, all vectors with entries in $A$ have $G(D, s) \cdot v=0$, and all functions $F(D) \rightarrow A$ lie in $\mathcal{D}_{A}(D)$. We proceed with the assumption that there is at least one crossing in $D$.

Let $S$ be a shaded face of $D$. If $S$ does not share a crossing of $D$ with any other shaded face then the value of $d(S)$ is handled by the first sentence of the second paragraph (as modified in the third paragraph). In this case it is obvious that $d(S)$ is well defined. Proposition 9 tells us that $v_{i}=v_{j}$ whenever $F_{i}$ and $F_{j}$ share a crossing of $D$ with $S$, so $d$ satisfies Definition 2 at all crossings involving $S$. If all shaded faces of $D$ are of this type, we are done.

The rest of the proof resembles the proof of Theorem 4 . Suppose $S$ is a shaded face such that $d(S)$ is defined through the process of the second paragraph, by applying Definition 2 at a crossing where $S$ and another shaded face are incident. Let $S_{0}, c_{1}, S_{1}, \ldots, c_{k}, S_{k}=S$ be the sequence of shaded faces and crossings that was used to determine the value of $d(S)$, with $S_{0}$ handled by the first sentence of the second paragraph. This sequence corresponds to a piecewise smooth path $P$ from a point in the interior of $S_{0}$ near $c_{1}$ to a point in the interior of $S$ near $c_{k}$, which stays inside shaded faces except when it passes through crossings. Any other possible recursive definition of $d(S)$ corresponds to a similar path $P^{\prime}$ from $S_{0}$ to $S$, which can be deformed into $P$ by some sequence of two types of moves: the type illustrated in Figure 6, and the trivial type in which two consecutive passages in opposite directions through the same crossing are canceled. 


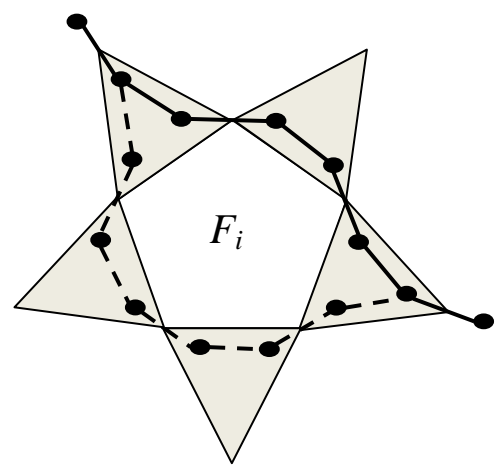

Figure 6: Deforming $P$.

To verify that $d(S)$ is well defined, we show that the deformation illustrated in Figure 6 does not change the value of $d(S)$. Let $S_{p}, c_{p+1}, S_{p+1}, \ldots, c_{q}, S_{q}$ be the portion of the sequence $S_{0}, c_{1}, S_{1}, \ldots, c_{k}, S_{k}$ that is a sequence of shaded neighbors of $F_{i}$. Then $P^{\prime}$ is obtained by replacing this portion with $S_{p}=$ $S_{p}^{\prime}, c_{p+1}^{\prime}, S_{p+1}^{\prime}, \ldots, c_{q^{\prime}}^{\prime}, S_{q^{\prime}}^{\prime}=S_{q}$, where the crossings $c_{p+1}, \ldots, c_{q}, c_{q+1}^{\prime}, \ldots, c_{p+1}^{\prime}$ appear in this order on a closed curve $\gamma$ contained in the boundary of $F_{i}$. Interchanging the names of $P$ and $P^{\prime}$ if necessary, we may presume that $c_{p+1}, \ldots, c_{q}$, $c_{q+1}^{\prime}, \ldots, c_{p+1}^{\prime}$ appear in this order clockwise around $\gamma$. For $1 \leq j \leq q-p$ let $U_{j}$ be the unshaded face that shares the crossing $c_{p+j}$ with $F_{i}$, and for $1 \leq j \leq q^{\prime}-p$ let $U_{j}^{\prime}$ be the unshaded face that shares the crossing $c_{p+j}^{\prime}$ with $F_{i}$. Then when the second paragraph states that at each step the unique value of $d\left(U_{j}\right)$ or $d\left(U_{j}^{\prime}\right)$ that satisfies Definition 2 is used, it means that for each $j \geq 1$,

$$
\begin{aligned}
& \qquad \begin{aligned}
d\left(S_{p+j}\right)= & d\left(S_{p+j-1}\right)+\eta\left(c_{p+j}\right) \cdot\left(d\left(U_{j}\right)-d\left(F_{i}\right)\right) \\
= & d\left(S_{p+j-1}\right)+\eta\left(c_{p+j}\right) \cdot\left(v\left(U_{j}\right)-v\left(F_{i}\right)\right)
\end{aligned} \\
& \text { and } d\left(S_{p+j}^{\prime}\right)=d\left(S_{p+j-1}^{\prime}\right)-\eta\left(c_{p+j}^{\prime}\right) \cdot\left(d\left(U_{j}^{\prime}\right)-d\left(F_{i}\right)\right) \\
& =d\left(S_{p+j-1}^{\prime}\right)-\eta\left(c_{p+j}^{\prime}\right) \cdot\left(v\left(U_{j}^{\prime}\right)-v\left(F_{i}\right)\right) .
\end{aligned}
$$

When we follow $P$ we conclude that

$$
d\left(S_{q}\right)-d\left(S_{p}\right)=\sum_{j=1}^{q-p}\left(d\left(S_{p+j}\right)-d\left(S_{p+j-1}\right)\right)=\sum_{j=1}^{q-p} \eta\left(c_{p+j}\right) \cdot\left(v\left(U_{j}\right)-v\left(F_{i}\right)\right)
$$

and when we follow $P^{\prime}$, we conclude that

$$
d\left(S_{q^{\prime}}^{\prime}\right)-d\left(S_{p}\right)=\sum_{j=1}^{q^{\prime}-p}\left(d\left(S_{p+j}^{\prime}\right)-d\left(S_{p+j-1}^{\prime}\right)\right)=-\sum_{j=1}^{q^{\prime}-p} \eta\left(c_{p+j}^{\prime}\right) \cdot\left(v\left(U_{j}^{\prime}\right)-v\left(F_{i}\right)\right) .
$$


The difference between these two values is

$$
\begin{gathered}
-\sum_{j=1}^{q-p} \eta\left(c_{p+j}\right) \cdot\left(v\left(U_{j}\right)-v\left(F_{i}\right)\right)-\sum_{j=1}^{q^{\prime}-p} \eta\left(c_{p+j}^{\prime}\right) \cdot\left(v\left(U_{j}^{\prime}\right)-v\left(F_{i}\right)\right) \\
=\left(\sum_{j=1}^{q-p} \eta\left(c_{p+j}\right)+\sum_{j=1}^{q^{\prime}-p} \eta\left(c_{p+j}^{\prime}\right)\right) \cdot v\left(F_{i}\right) \\
-\sum_{j=1}^{q-p} \eta\left(c_{p+j}\right) \cdot v\left(U_{j}\right)-\sum_{j=1}^{q^{\prime}-p} \eta\left(c_{p+j}^{\prime}\right) \cdot v\left(U_{j}^{\prime}\right)=\rho(\gamma) \cdot v
\end{gathered}
$$

where $\rho(\gamma)$ is the vector discussed in Proposition 8, As $\rho(\gamma) \cdot v=0, P$ and $P^{\prime}$ lead to the same value for $d(S)$.

It remains to verify that $d \in \mathcal{D}_{A}(D)$. Suppose $c$ is a crossing of $D$, as pictured in Figure 1. If a single shaded face $S$ appears twice in the figure then Definition 2 requires that the two unshaded faces $F_{i}, F_{j}$ in the figure have the same $d$ value. Proposition 9 assures us that this is the case. If two different shaded faces appear, then the well-definedness of $d$ allows us to assume that the $d$ value of one of the two shaded faces is calculated directly from the $d$ value of the other one, as in the third sentence of the second paragraph of the proof. But this calculation is performed precisely to guarantee that $d$ satisfies Definition 2 at the crossing $c$.

Combining Propositions 10 and 11, we obtain the following.

Theorem 12 Let $D$ be a link diagram with a shading s, and $A$ an abelian group. Then $v: \mathcal{D}_{A}(D) \rightarrow \operatorname{ker}_{A} G(D, s)$ is a surjective homomorphism, whose kernel consists of the Dehn colorings that are identically 0 on unshaded faces of $D$.

The next definition allows us to give a precise description of ker $v$.

Definition 13 Let $D$ be a link diagram with a shading $s$. Let $\Gamma_{s}(D)$ denote the shaded checkerboard graph of $D$, i.e., $\Gamma_{s}(D)$ has a vertex for each shaded face of $D$ and an edge for each crossing of $D$, with the edge corresponding to a crossing $c$ incident on the vertex (or vertices) corresponding to the shaded face $(s)$ incident at $c$. Then $\beta_{s}(D)$ denotes the number of connected components of $\Gamma_{s}(D)$.

If $d \in \operatorname{ker} v$ then $d$ is identically 0 on unshaded faces of $D$, so Definition 2 is equivalent to the requirement that $d(S)=d\left(S^{\prime}\right)$ whenever $S$ and $S^{\prime}$ are shaded faces of $D$ incident at the same crossing. It follows that $d$ is constant on each connected component of $\Gamma_{s}(D)$, and these constant values are arbitrary. We deduce that

$$
\operatorname{ker} v \cong A^{\beta_{s}(D)} .
$$




\section{The theorem of Nanyes}

In this section we discuss two versions of the theorem of Nanyes [20], one involving Dehn colorings and the other involving Fox colorings. The first version asserts that like $\varphi: \mathcal{D}_{A}(D) \rightarrow \mathcal{F}_{A}(D)$, the epimorphism $v: \mathcal{D}_{A}(D) \rightarrow$ $\operatorname{ker}_{A} G(D, s)$ splits.

Theorem 14 Let $D$ be a link diagram, and $A$ an abelian group. Then $\mathcal{D}_{A}(D)$ is the internal direct sum of ker $v$ and a subgroup isomorphic to $\operatorname{ker}_{A} G(D, s)$.

Proof. Choose shaded faces $S_{1}, \ldots, S_{\beta_{s}(D)}$ of $D$, one in each connected component of the graph $\Gamma_{s}(D)$. Let $u: \operatorname{ker}_{A} G(D, s) \rightarrow \mathcal{D}_{A}(D)$ be the map defined by the construction in the proof of Proposition 11, always using one of $S_{1}, \ldots, S_{\beta_{s}(D)}$ when implementing the first sentence of the second paragraph, and always using 0 for the value of $d\left(S_{i}\right)$. The uniqueness of the $d$ values calculated in the other steps of the recursion guarantees that $u$ is a homomorphism. As $v u$ is the identity map of $\operatorname{ker}_{A} G(D, s)$, the theorem follows.

Theorem 14 implies that

$$
\mathcal{D}_{A}(D) \cong \operatorname{ker}_{A} G(D, s) \oplus A^{\beta_{s}(D)} .
$$

The second version of the theorem of Nanyes 20] is the corresponding description of $\mathcal{F}_{A}(D)$ up to isomorphism. (N.b. If $A$ is an abelian group then $A^{0}$ denotes $\{0\}$, the abelian group with only one element.)

Theorem 15 Let $D$ be a link diagram, and $A$ an abelian group. Then

$$
\mathcal{F}_{A}(D) \cong \operatorname{ker}_{A} G(D, s) \oplus A^{\beta_{s}(D)-1}
$$

Proof. Choose a shaded face $S_{1}$ of $D$, and let

$$
\mathcal{D}_{1}=\left\{d \in \mathcal{D}_{A}(D) \mid d\left(S_{1}\right)=0\right\}
$$

If we apply the construction in the proof of Theorem 4 with $S_{1}$ always playing the role of $F_{0}$ and 0 always playing the role of $\alpha_{0}$, we conclude that the restricted mapping $\left(\varphi \mid \mathcal{D}_{1}\right): \mathcal{D}_{1} \rightarrow \mathcal{F}_{A}(D)$ is surjective. As $\operatorname{ker} \varphi=\left\{d_{\alpha,-\alpha} \mid \alpha \in A\right\}$, $\operatorname{ker}\left(\varphi \mid \mathcal{D}_{1}\right)=\{0\}$. Hence $\mathcal{F}_{A}(D) \cong \mathcal{D}_{1}$.

If we apply the construction in the proof of Proposition 11 with $S_{1}$ always used in the first implementation of the first sentence of the second paragraph and 0 always used as the arbitrarily chosen value of $d\left(S_{1}\right)$, then we conclude that the restricted mapping $\left(v \mid \mathcal{D}_{1}\right): \mathcal{D}_{1} \rightarrow \operatorname{ker}_{A} G(D, s)$ is surjective. The description of $\operatorname{ker} v$ at the end of the preceding section applies to $\operatorname{ker}\left(v \mid \mathcal{D}_{1}\right)$, with the exception that for $d \in \operatorname{ker}\left(v \mid \mathcal{D}_{1}\right)$ the value of $d$ on the connected component of $\Gamma_{s}(D)$ containing $S_{1}$ is not arbitrary; it is 0 . We deduce that $\operatorname{ker}\left(v \mid \mathcal{D}_{1}\right) \cong A^{\beta_{s}(D)-1}$.

Applying the proof of Theorem 14 to $\mathcal{D}_{1}$, we conclude that $\mathcal{D}_{1}$ is the internal direct sum of $\operatorname{ker}\left(v \mid \mathcal{D}_{1}\right)$ and a subgroup isomorphic to $\operatorname{ker}_{A} G(D, s)$. 
Notice that checkerboard colorings are not mentioned in Definitions 1 and 2 , but Theorems 14 and 15 tell us that $\mathcal{D}_{A}(D)$ and $\mathcal{F}_{A}(D)$ are determined up to isomorphism by the checkerboard graphs of $D$ : the unshaded checkerboard graph provides $G(D, s)$, and the shaded checkerboard graph provides $\beta_{s}(D)$. Although the two checkerboard graphs play different roles, the theorems apply equally well if the shading $s$ is reversed.

We should explain that we refer to Theorems 14 and 15 as "versions" of the theorem of Nanyes [20] because there are several differences between our setup and Nanyes's: he required the image of a Fox coloring to generate $A$, he required a Fox coloring to be 0 somewhere, and he removed a row and column from the Goeritz matrix. The first difference has the effect of shifting attention from the kernel to the cokernel of a homomorphism represented by $G(D, s)$. The second difference has the effect of removing a direct summand isomorphic to $A$ from $\mathcal{F}_{A}(D)$, and the third difference has the effect of removing another such direct summand from $\operatorname{ker}_{A} G(D, s)$. We leave further articulation of the details of the relationship between our discussion and that of 20] to the reader.

We should also point out that Nanyes's descriptions of $\beta_{s}(D)$ and $G(D, s)$ in 20] are inaccurate. He described $G(D, s)$ as a matrix obtained using all the faces of $D$, not just the unshaded faces; and he described $\beta_{s}(D)$ as the number of connected components of the graph of unshaded faces, not the graph of shaded faces. It is easy to see that either of these mistakes can lead to an erroneous description of $\mathcal{F}_{A}(D)$. For instance, let $D$ be a crossing-free diagram of a $\mu$ component unlink. Then $1 \leq \beta_{s}(D) \leq \mu$ and $D$ has $n=\mu+1-\beta_{s}(D)$ unshaded faces. The corresponding Goeritz matrix has all entries 0 , so if we mistakenly replace $G(D, s)$ with a matrix that has a row and column for every face, we will conclude that $\operatorname{ker}_{A} G(D, s) \oplus A^{\beta_{s}(D)-1}$ is isomorphic to $A^{n+2 \beta_{s}(D)-1}=$ $A^{\mu+\beta_{s}(D)}$. If we use the correct definition of $G(D, s)$ then we obtain the 0 matrix of order $n$, so $\operatorname{ker}_{A} G(D, s) \cong A^{n}$; if we then mistakenly calculate $\beta_{s}(D)$ using unshaded faces we will conclude that $\operatorname{ker}_{A} G(D, s) \oplus A^{\beta_{s}(D)-1}$ is isomorphic to $A^{2 n-1}$. The correct calculation yields $\operatorname{ker}_{A} G(D, s) \oplus A^{\beta_{s}(D)-1} \cong A^{n+\beta_{s}(D)-1}=$ $A^{\mu}$. This is isomorphic to $\mathcal{F}_{A}(D)$ because a Fox coloring of $D$ is simply a function that is constant on each component of $L(D)$.

\section{Two examples}

Let $T$ be the $(2,8)$ torus link diagram pictured on the left in Figure 7 , and $W$ the Whitehead link diagram pictured on the right. It is easy to see that $L(T)$ and $L(W)$ are inequivalent links: the linking number of the two components of $L(T)$ is \pm 4 , and the linking number of the two components of $L(W)$ is 0 .

Nevertheless, Theorems 14 and 15 imply that every abelian group $A$ has $\mathcal{D}_{A}(T) \cong \mathcal{D}_{A}(W)$ and $\mathcal{F}_{A}(T) \cong \mathcal{F}_{A}(W)$. To see why, notice that according to Definition 6 the Goeritz matrices of $T$ and $W$ associated with the shadings 

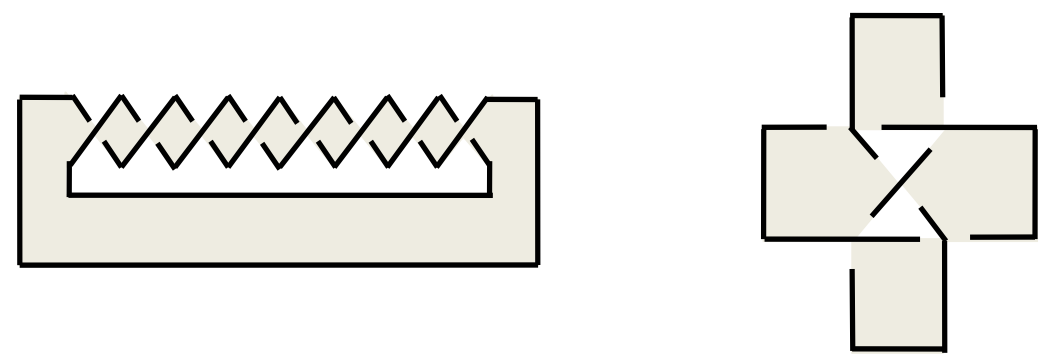

Figure 7: Diagrams of a $(2,8)$ torus link and a Whitehead link.

of Figure 7 are

$$
G(T, s)=\left(\begin{array}{cc}
-8 & 8 \\
8 & -8
\end{array}\right) \quad \text { and } \quad G(W, s)=\left(\begin{array}{ccc}
-3 & 1 & 2 \\
1 & -3 & 2 \\
2 & 2 & -4
\end{array}\right)
$$

Consequently, if $A$ is an abelian group then $\operatorname{ker}_{A} G(T, s)$ is the set of ordered pairs $(x, y) \in A^{2}$ such that $8 y-8 x=0$, and $\operatorname{ker}_{A} G(W, s)$ is the set of ordered triples $(a, b, c) \in A^{3}$ such that $-3 a+b+2 c=0$ and $a-3 b+2 c=0$.

We claim that for every abelian group $A$, the formula $\pi(a, b, c)=(a, c)$ defines an isomorphism $\pi: \operatorname{ker}_{A} G(W, s) \rightarrow \operatorname{ker}_{A} G(T, s)$. The claim is verified in four steps. First, notice that if $(a, b, c) \in \operatorname{ker}_{A} G(W, s)$ then

$$
8 c-8 a=3 \cdot(-3 a+b+2 c)+a-3 b+2 c=3 \cdot 0+0=0,
$$

so $\pi(a, b, c)=(a, c)$ is an element of $\operatorname{ker}_{A} G(T, s)$. Second, notice that $\pi$ is a homomorphism because it is a restriction of the projection homomorphism $A^{3} \rightarrow A^{2}$ defined by $(a, b, c) \mapsto(a, c)$. Third, notice that if $(x, y) \in \operatorname{ker}_{A} G(T, s)$ then $(x, 3 x-2 y, y) \in \operatorname{ker}_{A} G(W, s)$, because

$$
-3 x+(3 x-2 y)+2 y=0 \quad \text { and } \quad x-3 \cdot(3 x-2 y)+2 y=-8 x+8 y=0 .
$$

As $\pi(x, 3 x-2 y, y)=(x, y)$, we deduce that $\pi$ is surjective. Fourth, notice that if $(a, b, c),\left(a^{\prime}, b^{\prime}, c^{\prime}\right) \in \operatorname{ker}_{A} G(W, s)$ and $\pi(a, b, c)=\pi\left(a^{\prime}, b^{\prime}, c^{\prime}\right)$ then $a=a^{\prime}$ and $c=c^{\prime}$, so $b=3 a-2 c=3 a^{\prime}-2 c^{\prime}=b^{\prime}$. We deduce that $\pi$ is injective.

If $\bar{s}$ denotes the shadings of $T$ and $W$ opposite to those indicated in Figure 7. then $\beta_{s}(T)=\beta_{s}(W)=\beta_{\bar{s}}(T)=\beta_{\bar{s}}(W)=1$. According to Theorem 15, it follows that $\operatorname{ker}_{A} G(T, s) \cong \operatorname{ker}_{A} G(T, \bar{s})$ and $\operatorname{ker}_{A} G(W, s) \cong \operatorname{ker}_{A} G(W, \bar{s})$. We leave it as an exercise for the reader to verify these isomorphisms directly.

\section{$7 \quad$ A special case}

Several authors have observed that when $A=\mathbb{Z} / 2 \mathbb{Z}$, there is an especially simple relationship between $\operatorname{ker}_{A} G(D, s)$ and the link $L(D)$ [3, 9, 15, 20, 24, 25]. In 
fact this simple relationship holds more generally when the exponent of $A$ is 2, i.e., $2 \cdot \alpha=0 \forall \alpha \in A$. We close with a summary of this simple relationship.

Let $D$ be a link diagram, with $L(D)=K_{1} \cup \cdots \cup K_{\mu}$. For each $F \in F(D)$ there is a piecewise smooth path $P_{F}$ from a point in the interior of $F$ to a point in the interior of the unbounded face of $D$, which does not come near any crossing of $D$ and has only a finite number of intersections with the $\operatorname{arcs}$ of $D$, all of which are transverse intersections. In general there are many such paths, with different patterns of intersections with $D$; but every such path will have the same number of intersections $(\bmod 2)$ with the image of each $K_{i}$.

Definition 16 If $F \in F(D)$ then for $1 \leq i \leq \mu$ the index of $F$ with respect to $K_{i}$ is the parity $(\bmod 2)$ of the number of intersection points of a path $P_{F}$ with the image of $K_{i}$ in the plane. We denote the index $i_{D}\left(F, K_{i}\right)$.

Proposition 17 Suppose $A$ is of exponent 2. Then $f: A(D) \rightarrow A$ is a Fox coloring of $D$ if and only if there is an element $\left(\alpha_{1}, \ldots, \alpha_{\mu}\right) \in A^{\mu}$ such that $f(a)=\alpha_{i}$ whenever $a$ is an arc of $D$ that belongs to the image of $K_{i}$.

Proof. As $A$ is of exponent 2, Definition 1 simply requires $f\left(a_{1}\right)=f\left(a_{2}\right)$ in Figure 1. Applying this equation at every crossing, we conclude that $\mathcal{F}_{A}(D)$ is the set of maps $A(D) \rightarrow A$ that are constant on the image of every $K_{i}$.

That is, $\mathcal{F}_{A}(D)$ may be identified naturally with $A^{\mu}$. It follows from Theorem 5 that $\mathcal{D}_{A}(D)$ may be identified naturally with $A^{\mu+1}$. We spell out the details:

Proposition 18 Suppose $A$ is of exponent 2. Then $d: F(D) \rightarrow A$ is a Dehn coloring of $D$ if and only if there is an element $\left(\alpha_{0}, \ldots, \alpha_{\mu}\right) \in A^{\mu+1}$ such that every $F \in F(D)$ has

$$
d(F)=\alpha_{0}+\sum_{i=1}^{\mu} \alpha_{i} \cdot i_{D}\left(F, K_{i}\right) .
$$

Proof. The formula in the statement is the appropriate version of formula (1) from the proof of Theorem 4 .

Corollary 19 Suppose $A$ is of exponent 2 , and $F_{1}, \ldots, F_{n}$ are the unshaded faces of a shading $s$ of $D$. Then an element $v=\left(v_{1}, \ldots, v_{n}\right) \in A^{n}$ is contained in $\operatorname{ker}_{A} G(D, s)$ if and only if there is an element $\left(\alpha_{0}, \ldots, \alpha_{\mu}\right) \in A^{\mu+1}$ such that

$$
v_{j}=\alpha_{0}+\sum_{i=1}^{\mu} \alpha_{i} \cdot i_{D}\left(F_{j}, K_{i}\right)
$$

for every $j \in\{1, \ldots, n\}$.

Proof. According to Proposition 11, the elements of $\operatorname{ker}_{A} G(D, s)$ are the elements of $A^{n}$ obtained by evaluating Dehn colorings on the unshaded faces of D. 
The same formula appears in Proposition 18 and Corollary 19, but the two maps defined by the formula are quite different. In Proposition 18, the formula defines an isomorphism $A^{\mu+1} \rightarrow \mathcal{D}_{A}(D)$. In Corollary 19 instead, the formula defines a split epimorphism $A^{\mu+1} \rightarrow \operatorname{ker}_{A} G(D, s)$, whose kernel is isomorphic to $A^{\beta_{s}(D)}$.

\section{References}

[1] Colin C. Adams. The knot book. American Mathematical Society, Providence, RI, 2004. An elementary introduction to the mathematical theory of knots, Revised reprint of the 1994 original.

[2] J. Scott Carter, Daniel S. Silver, and Susan G. Williams. Three dimensions of knot coloring. Amer. Math. Monthly, 121(6):506-514, 2014.

[3] ZhiYun Cheng and HongZhu Gao. On region crossing change and incidence matrix. Sci. China Math., 55(7):1487-1495, 2012.

[4] Richard H. Crowell. Nonalternating links. Illinois J. Math., 3:101-120, 1959.

[5] Richard H. Crowell and Ralph H. Fox. Introduction to knot theory. Springer-Verlag, New York-Heidelberg, 1977. Reprint of the 1963 original, Graduate Texts in Mathematics, No. 57.

[6] Chris Godsil and Gordon Royle. Algebraic graph theory, volume 207 of Graduate Texts in Mathematics. Springer-Verlag, New York, 2001.

[7] Lebrecht Goeritz. Knoten und quadratische Formen. Math. Z., 36(1):647654, 1933.

[8] C. McA. Gordon and R. A. Litherland. On the signature of a link. Invent. Math., 47(1):53-69, 1978.

[9] Megumi Hashizume. On the homomorphism induced by region crossing change. JP J. Geom. Topol., 14(1):29-37, 2013.

[10] Young Ho Im, Kyeonghui Lee, and Sang Youl Lee. Signature, nullity and determinant of checkerboard colorable virtual links. J. Knot Theory Ramifications, 19(8):1093-1114, 2010.

[11] François Jaeger. On the Kauffman polynomial of planar matroids. In Fourth Czechoslovakian Symposium on Combinatorics, Graphs and Complexity (Prachatice, 1990), volume 51 of Ann. Discrete Math., pages 117127. North-Holland, Amsterdam, 1992.

[12] L. H. Kauffman. Remarks on Formal Knot Theory. arxiv:math/0605622, May 2006. 
[13] Louis H. Kauffman. Knots and physics, volume 53 of Series on Knots and Everything. World Scientific Publishing Co. Pte. Ltd., Hackensack, NJ, fourth edition, 2013.

[14] R. H. Kyle. Branched covering spaces and the quadratic forms of links. Ann. of Math. (2), 59:539-548, 1954.

[15] Kalyn R. Lamey, Daniel S. Silver, and Susan G. Williams. Vertex-colored graphs, bicycle spaces and Mahler measure. J. Knot Theory Ramifications, 25(6):1650033, 22, 2016.

[16] W. B. Raymond Lickorish. An introduction to knot theory, volume 175 of Graduate Texts in Mathematics. Springer-Verlag, New York, 1997.

[17] Magnhild Lien and William Watkins. Dual graphs and knot invariants. Linear Algebra Appl., 306(1-3):123-130, 2000.

[18] A. S. Lipson. Link signature, Goeritz matrices and polynomial invariants. Enseign. Math. (2), 36(1-2):93-114, 1990.

[19] Charles Livingston. Knot theory, volume 24 of Carus Mathematical Monographs. Mathematical Association of America, Washington, DC, 1993.

[20] Ollie Nanyes. Link colorability, covering spaces and isotopy. J. Knot Theory Ramifications, 6(6):833-849, 1997.

[21] Józef H. Przytycki. From Goeritz matrices to quasi-alternating links. In The mathematics of knots, volume 1 of Contrib. Math. Comput. Sci., pages 257-316. Springer, Heidelberg, 2011.

[22] K. Reidemeister. Knotentheorie. Springer-Verlag, Berlin-New York, 1974. Reprint.

[23] H. Seifert. Die Verschlingungsinvarianten der zyklischen Knotenüberlagerungen. Abh. Math. Sem. Univ. Hamburg, 11(1):84-101, 1935.

[24] Daniel S. Silver and Susan G. Williams. On the component number of links from plane graphs. J. Knot Theory Ramifications, 24(1):1520002, 5, 2015.

[25] Lorenzo Traldi. On the Goeritz matrix of a link. Math. Z., 188(2):203-213, 1985. 